\title{
Purpura Fulminans in Infantile Streptococcal Septicemia
}

\author{
Saurabh Piparsania ${ }^{1}$, Nitin Rajput ${ }^{1}$, Kuldeep Singh ${ }^{1}$, Priti Zade ${ }^{1}$, Milind Joshi ${ }^{2 *}$ \\ ${ }^{1}$ Department of Paediatrics, Sri Aurobindo Institute of Medical Sciences, Indore, India \\ ${ }^{2}$ Department of Paediatric surgery, Sri Aurobindo Institute of Medical Sciences, Indore, India \\ E-mail: *milindj79@yahoo.com \\ Received June 12, 2011; revised September 15, 2011; accepted October 14, 2011
}

\begin{abstract}
Purpura fulminans is a hemorrhagic condition associated predominantly with meningococcal and other gram negative septicemias. It occurs mainly in infants and younger children. Features include tissue necrosis, small vessel thrombosis, disseminated intravascular coagulation, multi-organ failure and death. Other causes include clotting factor deficiencies and idiopathic varieties. The condition is uncommon due to gram positive bacterial sepsis. We report one such case with gram positive bacterial infection.
\end{abstract}

Keywords: Purpura Fulminans, Gram Positive Sepsis

\section{Introduction}

Purpura fulminans is a hemorrhagic condition associated predominantly with meningococcal and other gram negative septicemias. It occurs mainly in infants and younger children. Features include tissue necrosis, small vessel thrombosis, disseminated intravascular coagulation, multiorgan failure and death. Other causes include clotting factor deficiencies and idiopathic varieties. The condition is uncommon due to gram positive bacterial sepsis. We report a case of purpura fulminans caused by alphahemolytic streptococcal sepsis and meningitis in a 4 month old baby girl who was referred to our institute with digital gangrene and necrotic and hemorrhagic purpurae.

\section{Case Report}

A 4 month old, baby girl was referred to our institute with digital gangrene (Figure 1) and hemorrhagic and necrotic purpurae (Figure 2). She had presented 3 days back to her local hospital with high grade fever, poor feeding, drowsiness and generalized tonic-clonic seizures. Erythematous rash had first appeared over her abdomen with gradual spread to involve trunk and thighs. A diagnosis of probable meningococcal septicemia was made and baby was treated accordingly. Due to rapid deterioration in the condition of the patient over the next 2 - 3 days, she was referred to our institute for further management.

On examination, toxic, febrile child with features of meningitis and septic shock were present. Hemorrhagic and necrotic purpurae were seen all over the body with digital gangrene.

Laboratory investigation revealed raised white cell counts (39,700/cu mm) with neutrophilia (80\%). Platelet count was 40,000/cu mm. Blood and CSF cultures were positive for alpha-hemolytic streptococci. Both PT and aPTT were prolonged. Levels of fibrin degradation products were elevated. Protein C and S levels were normal.

Baby was managed with antibiotics, IV fresh frozen plasma, platelet concentrate, LMW heparin, inotropes, mechanical ventilation and other supportive measures. Baby improved clinically over next 5 - 6 days. At this stage, no new purpurae were appearing and the older ones regressed. (Figure 3) Supportive treatment was continued, and patient was discharged after 14 days of hospital stay.

\section{Discussion}

Purpura fulminans (PF) was first described by Guelliot in 1884 [1]. This is a hemorrhagic condition usually associated with septicemic conditions. Features include high grade fever, low blood pressure, disseminated intravascular coagulation (DIC) and purpura leading to tissue necrosis with small vessel thrombosis. Digital gangrene is not very common. The condition is more common with meningococcal and other gram negative septicemias [2] and uncommon with gram positive organisms.

$\mathrm{PF}$ is an acute onset disorder characterized by cutaneous hemorrhage and necrosis caused by DIC and dermal 


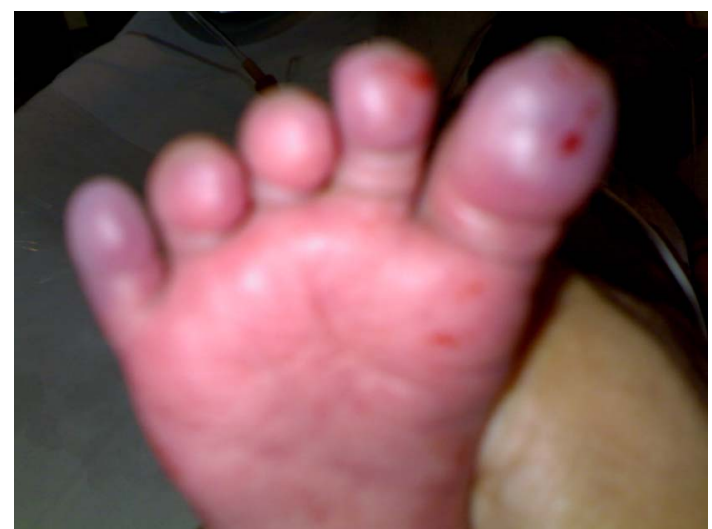

Figure 1. Clinical photograph showing digital gangrene.

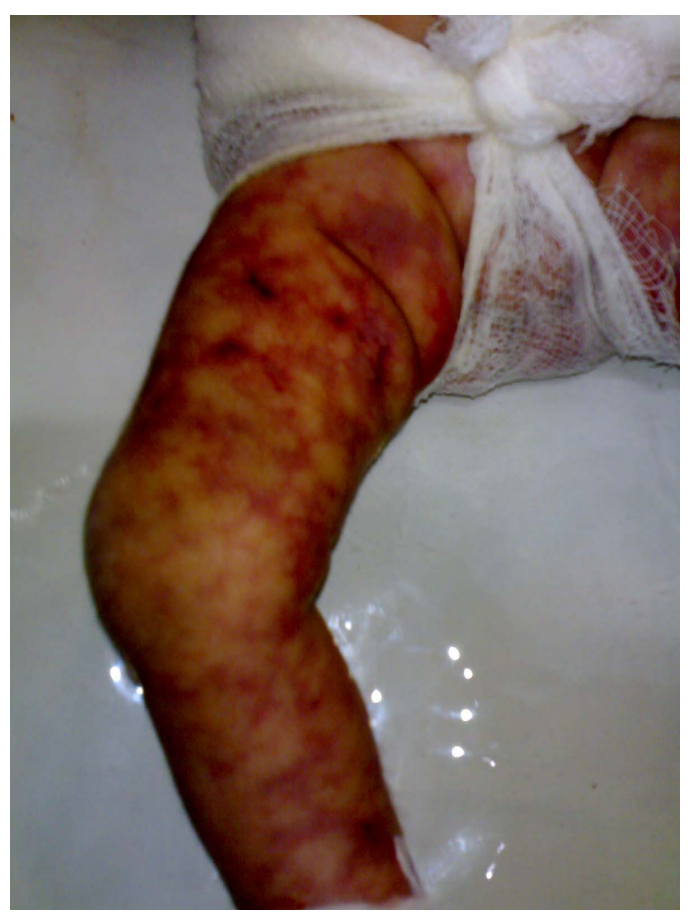

Figure 2. Clinical photograph showing extensive lesions of purpura fulminans.

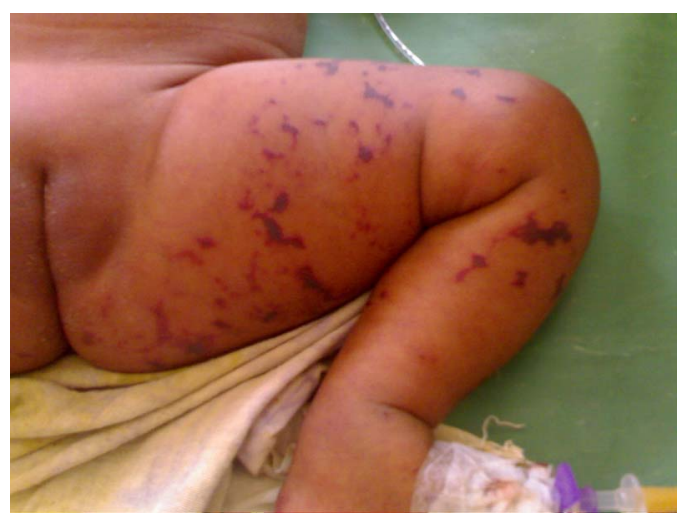

Figure 3. Clinical photographs showing regressing lesions in recovery phase. vascular thrombosis. Three distinct categories can be identified: inherited or acquired abnormalities of the protein $\mathrm{C}$ or other coagulation proteins, "acute infectious PF" and "idiopathic PF" [3].

Majority of the cases of purpura fulminans are thought to be caused by the inherited or acquired abnormalities of protein $\mathrm{C}$ and protein $\mathrm{S}$ anticoagulant pathway, whereas gram-negative organisms are still the commonest cause of the acute infectious variety [4].

Meningococcal septicemia is the most common acute infection with which purpura fulminans is associated, and the development of PF in this condition usually indicate poor prognosis [4]. It is also a common association with varicella and, less commonly with measles and pneumococcal sepsis. In the neonatal period group $\mathrm{B}$ streptococcus appears to be the major cause, other pathogens described include staphylococcus, Escherichia coli and Enterobacter.

The lesions of PF are differentiated from other purpuric lesions by their characteristic appearance. Initial dermal erythema and intense pain is rapidly replaced by painful, indurated, irregular blue-black central areas of hemorrhagic necrosis with a surrounding erythematous border [5]. Vesicles and bullae may form. Lesions are generally sterile, although secondary infection of gangrenous tissue is not uncommon. Necrosis may spread to involve underlying muscle and bone. Healing leads to scarring, and auto-amputation of digits and extremities may occur.

The idiopathic variety is largely confined to the skin and mainly involves the lower half of the body. A similar distribution is seen in those with protein $C$ or protein $S$ deficiency, whereas infectious necrosis often begins distally with proximal progression or diffuse spread over the body.

Differential diagnoses include thrombotic thrombocytopenic purpura Henoch-Schönlein purpura and post infectious thrombocytopenic purpura. However skin necrosis is not severe in any of these conditions.

Treatment is removal of the underlying cause and supportive with FFP, LMW heparin and adequate analgesia. Some patients require extensive surgical debridement and skin grafting. These patients usually have multiple intravascular thromboses complicationg the condition. In some cases digits may need to be amputated [2].

\section{References}

[1] W. D. James, T. G. Berger, et al., “Andrews’ Diseases of the Skin: Clinical Dermatology," Saunders Elsevier, Readfield, 2006.

[2] J. Nolan and R. Sinclair, "Review of Management of Purpura Fulminans and Two Case Reports,” British Journal of Anaesthesia, Vol. 86, No. 4, 2001, pp. 581-586. 
doi:10.1093/bja/86.4.581

[3] D. M. Adcock, J. Brozna and R. A. Marlar "Proposed Classification and Pathologic Mechanisms of Purpura Fulminans and Skin Necrosis," Seminars in Thrombosis and Hemostasis, Vol. 16, No. 4, 1990, pp. 333-340. doi:10.1055/s-2007-1002686

[4] G. L. Darmstadt, "Acute Infectious Purpura Fulminans:
Pathogenesis and Medical Management,” Pediatric Dermatology, Vol. 15, No. 3, 1998, pp. 169-183. doi:10.1046/j.1525-1470.1998.1998015169.x

[5] R. B. Francis, “Acquired Purpura Fulminans,” Seminars in Thrombosis and Hemostasis, Vol. 16, No. 4, 1990, pp. 310-325. doi:10.1055/s-2007-1002684 